\title{
Significant enhancement of critical current density by effective carbon-doping in $\mathrm{MgB}_{2}$ thin films
}

\author{
Mahipal Ranot, O. Y. Lee, and W. N. Kang* \\ BK21 Physics Division and Department of Physics, Sungkyunkwan University, Suwon, Korea
}

(Received 4 June 2013; revised or reviewed 26 June 2013; accepted 27 June 2013)

\begin{abstract}
The pure and carbon (C)-doped $\mathrm{MgB}_{2}$ thin films were fabricated on $\mathrm{Al}_{2} \mathrm{O}_{3}(0001)$ substrates at a temperature of $650{ }^{\circ} \mathrm{C}$ by using hot-filament-assisted hybrid physical-chemical vapor deposition technique. The $T_{c}$ value for pure $\mathrm{MgB}_{2}$ film is $38.5 \mathrm{~K}$, while it is between 30 and $35 \mathrm{~K}$ for carbon-doped $\mathrm{MgB}_{2}$ films. Expansion in $c$-axis lattice parameter was observed with increase in carbon doping concentration which is in contrast to carbon-doped $\mathrm{MgB}_{2}$ single crystals. Significant enhancement in the critical current density was obtained for C-doped $\mathrm{MgB}_{2}$ films as compared to the undoped $\mathrm{MgB}_{2}$ film. This enhancement is most probably due to the incorporation of $\mathrm{C}$ into $\mathrm{MgB}_{2}$ and the high density of grain boundaries, both help in the pinning of vortices and result in improved superconducting performance.
\end{abstract}

Keywords : $\mathrm{MgB}_{2}$ film, carbon doping, HFA-HPCVD

\section{INTRODUCTION}

The remarkably high transition temperature $\left(T_{c}=39 \mathrm{~K}\right)$ of $\mathrm{MgB}_{2}$ makes it practically more advantageous over conventional metallic superconductors [1]. The strongly linked nature of the intergrains with a high charge carrier density in this material makes it further an attractive candidate for the next generation of superconductor applications. For most of the practical applications, high critical current density $\left(J_{\mathrm{c}}\right)$ in the presence of a magnetic field is required [2]. The $J_{\mathrm{c}}$ of undoped $\mathrm{MgB}_{2}$ is high enough $10^{6}-10^{7} \mathrm{~A} / \mathrm{cm}^{2}$ at low magnetic fields for practical application, however, $J_{\mathrm{c}}$ drops rapidly with increasing magnetic field due to the low $H_{\mathrm{c} 2}$ and the lack of the effective pinning sites in $\mathrm{MgB}_{2}$ [3]. Therefore the improvement of $J_{\mathrm{c}}$ under magnetic field is indispensable for the development of $\mathrm{MgB}_{2}$ material for magnet applications. The substitution of carbon atoms into the boron sites of $\mathrm{MgB}_{2}$ are known to be the most effective way to improve pinning properties in $\mathrm{MgB}_{2}$ and hence $J_{\mathrm{c}}$ performance under high magnetic fields [4]. The current-carrying performance has been improved by carbon in all the form of $\mathrm{MgB}_{2}$ superconductors, such as polycrystalline bulk, single crystals, wires, tapes, filaments, fibers and thin films. Despite much research done on enhancement of $J_{\mathrm{c}}$ in $\mathrm{MgB}_{2}$ bulk samples, only a little work has been reported for improving $J_{\mathrm{c}}$ in $\mathrm{MgB}_{2}$ films [5]. Few reports have shown the improvement in $J_{\mathrm{c}}$ by means of carbon doping in $\mathrm{MgB}_{2}$ films $[6,7]$. Here, we are reporting the effect of carbon-doping on the superconducting properties of $\mathrm{MgB}_{2}$ thin films. The carbon-doped $\mathrm{MgB}_{2}$ films were fabricated

\footnotetext{
* Corresponding author: wnkang@skku.edu
}

by using hot-filament-assisted hybrid physical-chemical vapor deposition (HPCVD) using methane as the doping source.

\section{EXPERIMENTAL}

The carbon-doped $\mathrm{MgB}_{2}$ thin films were fabricated on $\mathrm{Al}_{2} \mathrm{O}_{3}(000 l)$ substrates by using a modified HPCVD system. Inspite of capable of producing high-quality $\mathrm{MgB}_{2}$ films, it is difficult to dope the $\mathrm{MgB}_{2}$ films using the original HPCVD system [3]. Therefore, additional dopant methane $\left(\mathrm{CH}_{4}\right)$ gas line was installed in the original HPCVD system for doping $\mathrm{MgB}_{2}$ thin films. A separate heater, a Kanthal-super filament, was also installed to decompose $\mathrm{CH}_{4}$ gas. In this hot-filament-assisted (HFA)-HPCVD process, the $\mathrm{Al}_{2} \mathrm{O}_{3}$ substrate was placed on the top surface of a susceptor and Mg chips were placed around it. The reactor was firstly evacuated to a base pressure of $\sim 10^{-3}$ Torr using rotary pump and purged several times by flowing high purity argon and hydrogen gases. Prior to the carbon-doped $\mathrm{MgB}_{2}$ film growth, the susceptor along with substrate and $\mathrm{Mg}$ chips were inductively heated towards the set temperature under a reactor pressure of 100 Torr in $\mathrm{H}_{2}$ atmosphere. Upon reaching the set temperature, a boron precursor gas, $\mathrm{B}_{2} \mathrm{H}_{6}$ $\left(5 \%\right.$ in $\left.\mathrm{H}_{2}\right)$ and $\mathrm{CH}_{4}(100 \%)$ gas were introduced into the reactor to initiate the film growth. Finally, the fabricated C-doped $\mathrm{MgB}_{2}$ film was cooled down to room temperature in a flowing $\mathrm{H}_{2}$ carrier gas. The flow rates were $100 \mathrm{sccm}$ for the $\mathrm{H}_{2}$ carrier gas and $50 \mathrm{sccm}$ for the $\mathrm{B}_{2} \mathrm{H}_{6} / \mathrm{H}_{2}$ mixture. $\mathrm{CH}_{4}$ gas of different flow rates was added to the carrier gas 
to dope the film with different carbon concentrations. All the carbon-doped $\mathrm{MgB}_{2}$ films were fabricated at a substrate temperature of $650{ }^{\circ} \mathrm{C}$. $\mathrm{CH}_{4}$ was decomposed by the hot filament at $900{ }^{\circ} \mathrm{C}$. A pure $\mathrm{MgB}_{2}$ film was also prepared under the same conditions for comparison. The nominal carbon concentrations were determined using the correlation between gas concentrations and flow rates. For example, for the flow rates of 50,100 , and $5 \mathrm{sccm}$ of $\mathrm{B}_{2} \mathrm{H}_{6}$, $\mathrm{H}_{2}$, and $\mathrm{CH}_{4}$, respectively, the calculated nominal carbon concentration is $3.2 \%$.

The crystal structures of pure and carbon-doped $\mathrm{MgB}_{2}$ films were investigated by X-ray diffraction (D8 discover, Bruker AXS) using $\mathrm{Cu} \mathrm{K} \alpha$ as an X-ray source. Scanning electron microscopy (SEM) was used for measuring the surface morphologies and the thicknesses of the films. The standard four-probe method was used to measure the temperature dependence of resistivity for all the prepared films. The magnetization hysteresis $(M-H)$ measurements were carried out using a magnetic property measurement system (XL-5S, Quantum Design).

\section{RESULTS AND DISCUSSION}

The X-ray $\theta-2 \theta$ scans of pure and carbon-doped $\mathrm{MgB}_{2}$ thin films with different nominal carbon concentrations of 2.6, 3.2, 3.8, and $5.0 \%$ are shown in Fig. 1a. The XRD patterns show only $(000 l)$ peaks of $\mathrm{MgB}_{2}$ indicating that the films are $c$-axis oriented. Expansion in $c$-axis lattice parameter was observed with increase in carbon doping concentration, which is similar to carbon-doped films [6] but in contrast to carbon-doped single crystals, where the $c$-axis remains almost constant for all the carbon concentrations [8]. Compared to the undoped film, the $\mathrm{MgB}_{2}(000 l)$ peaks are suppressed as carbon concentration increases. The magnification of $\mathrm{MgB}_{2}$ (0001) peak is shown in Fig. 1b. Moreover, there is no indication of any secondary phases, such as $\mathrm{Mg}, \mathrm{MgO}$, and $\mathrm{MgB}_{4}$.

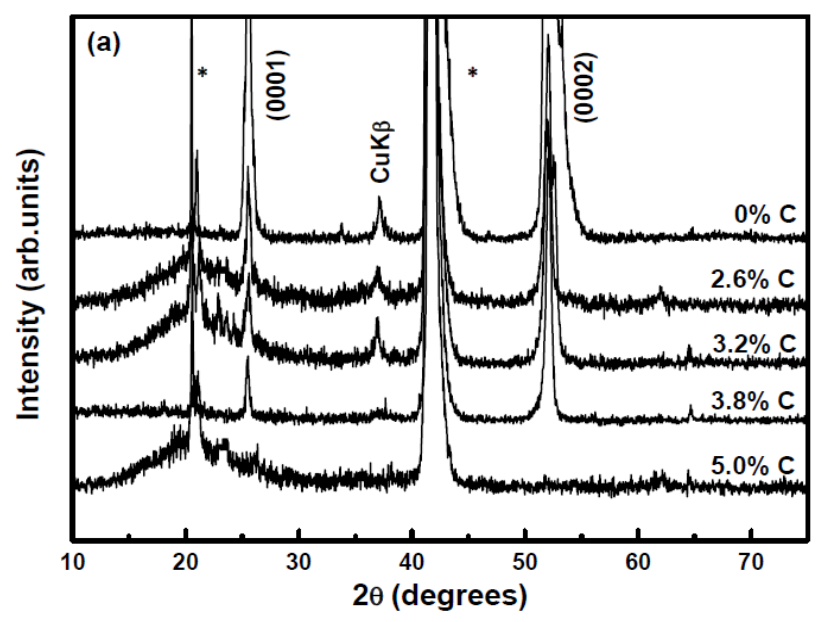

Fig. 1. (a) XRD patterns of pure and carbon-doped $\mathrm{MgB}_{2}$ thin films.

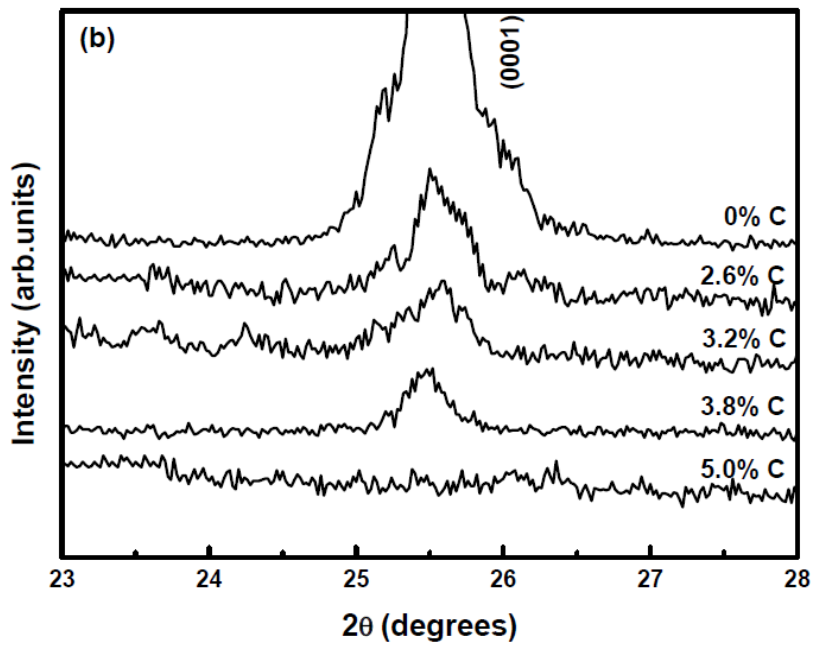

Fig. 1. (b) Magnified view of 0001 peak of $\mathrm{MgB}_{2}$, suppression of peak was observed as the doping concentration increases.

The resistivity versus temperature curves for pure and carbon-doped $\mathrm{MgB}_{2}$ films are plotted in Fig. 2. The inset shows the magnified view near the superconducting transition temperature $\left(T_{c}\right)$. The $T_{c}$ value for pure $\mathrm{MgB}_{2}$ film is $38.5 \mathrm{~K}$, while it is between 30 and $35 \mathrm{~K}$ for carbon-doped $\mathrm{MgB}_{2}$ films. The reduction in $T_{c}$ could also be an indicator of carbon substitution at boron sites of $\mathrm{MgB}_{2}$. The carbon-doped films exhibit a systematic increase in resistivity with increasing carbon concentration, except for $3.2 \% \mathrm{C}$-doped sample. The residual resistivity ratio $\left(\mathrm{RRR}=\rho_{300 \mathrm{~K}} / \rho_{40 \mathrm{~K}}\right)$ values for the pure and $\mathrm{C}$-doped films with concentrations of 2.6, 3.2, 3.8, and 5.0 \% are 4.3, $1.4,1.3,1.5$, and 1.4 respectively. It indicates that the impurity scattering is stronger in carbon-doped $\mathrm{MgB}_{2}$ films, and hence the decreased RRR values.

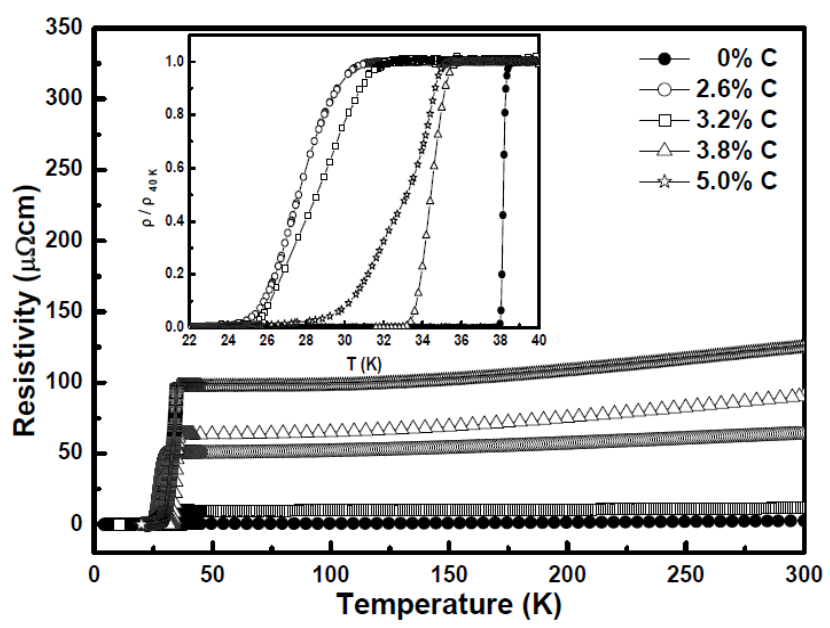

Fig. 2. Resistivity versus temperature curves of pure and carbon-doped $\mathrm{MgB}_{2}$ films with different nominal carbon concentrations of $2.6,3.2,3.8$, and $5.0 \%$. 


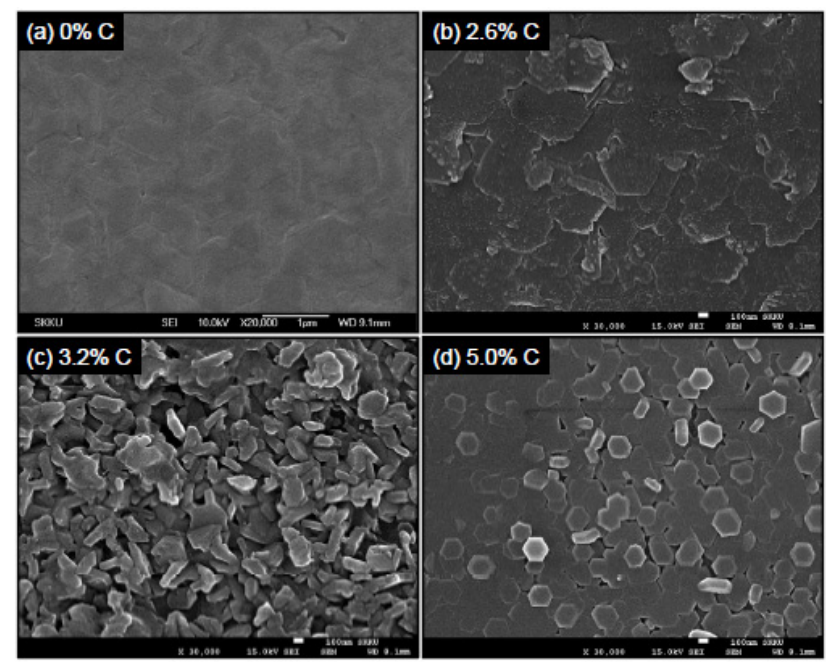

Fig. 3. SEM images of pure (a) and C-doped $\mathrm{MgB}_{2}$ films with different nominal carbon concentrations of (b) $2.6 \%$, (c) $3.2 \%$, and (d) $5.0 \%$.

The surface morphologies of pure and doped $\mathrm{MgB}_{2}$ films with different carbon concentrations are shown in Fig. $3 a-d$ The dense and smooth morphology was observed for pure $\mathrm{MgB}_{2}$ thin film. As the pure film was doped with carbon, the surface morphology changes from smooth to a planar structure for $2.6 \% \mathrm{C}$-doped $\mathrm{MgB}_{2}$ film. With further increase in carbon doping concentration, the average grain size was found to decrease from $450 \mathrm{~nm}$ for $2.6 \% \mathrm{C}$-doped film to $200 \mathrm{~nm}$ for $5.0 \% \mathrm{C}$-doped film. It indicates that the carbon doping suppresses the $\mathrm{MgB}_{2}$ grain growth and reduces the grain size. Both the carbon doping and the high density of grain boundaries which are known to be the main pinning source in $\mathrm{MgB}_{2}$, are expected to be beneficial for improving the $J_{\mathrm{c}}$ performance of $\mathrm{MgB}_{2}$ superconductor.

The critical current density $\left(J_{\mathrm{c}}\right)$ is evaluated from the magnetic hysteresis $(M-H)$ loops by using the Bean critical state model. Fig. 4 shows the magnetic field dependence of $J_{\mathrm{c}}$ at $5 \mathrm{~K}$ for pure and $\mathrm{C}$-doped $\mathrm{MgB}_{2}$ films. At self-field both the C-doped films show nearly comparable $J_{\mathrm{c}}$ to that of pure sample. This is in contrast to the reported data on C-doped $\mathrm{MgB}_{2}$ films, where one order of magnitude of reduction in $J_{\mathrm{c}}$ was noticed at self-field and $5 \mathrm{~K}$ [6]. It indicates that the inter-grain connectivity for $\mathrm{C}$-doped films is denser and comparable to the pure one. For the undoped film, $J_{\mathrm{c}}$ drops rapidly in the presence of magnetic field and it is $10^{2} \mathrm{~A} / \mathrm{cm}^{2}$ at an applied field of $2 \mathrm{~T}$. On the other hand, the $3.2 \% \mathrm{C}$-doped $\mathrm{MgB}_{2}$ film exhibits a $J_{\mathrm{c}}$ of the order of $10^{5} \mathrm{~A} / \mathrm{cm}^{2}$ at the corresponding field. Significantly high $J_{\mathrm{c}}$ values at high fields are obtained for the C-doped films. This enhancement in $J_{\mathrm{c}}$ is most likely due to the incorporation of $\mathrm{C}$ into $\mathrm{MgB}_{2}$ and the high density of grain boundaries, both help in the pinning of vertices and result in improved superconducting performance.

\section{CONCLUSION}

The effect of carbon-doing on microstructure and

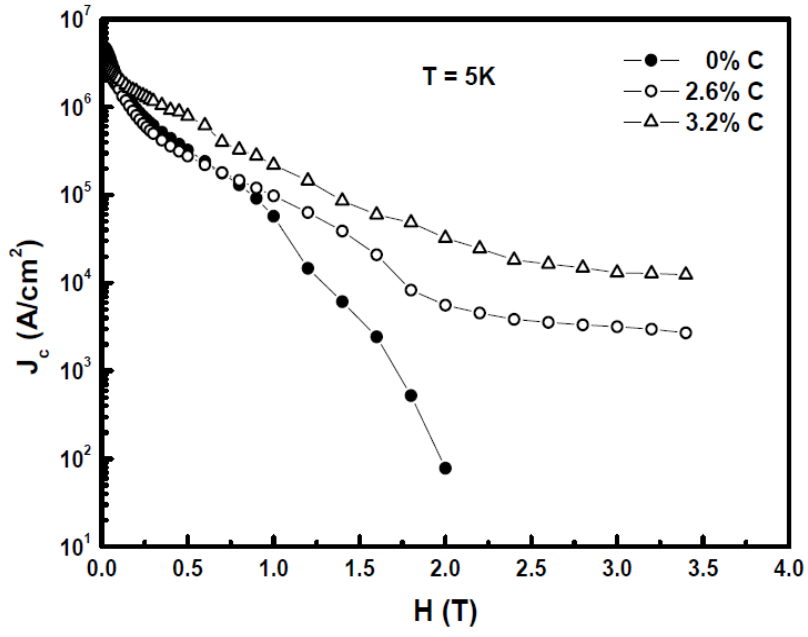

Fig. 4. $J_{\mathrm{c}}(H)$ curves at $5 \mathrm{~K}$ for pure and $\mathrm{C}$-doped $\mathrm{MgB}_{2}$ films with different nominal carbon concentrations of 2.6 and $3.2 \%$.

superconducting properties of $\mathrm{MgB}_{2}$ thin films were investigated. As compared to pure $\mathrm{MgB}_{2}$ film, reduction in $T_{c}$, decrease in RRR values and increase in resistivity were observed for $\mathrm{C}$-doped $\mathrm{MgB}_{2}$ films. The average grain size was found to decrease with increase in carbon doping concentration. It indicates that the carbon doping suppresses the $\mathrm{MgB}_{2}$ grain growth and reduces the grain size. At high magnetic fields the $\mathrm{C}$-doped $\mathrm{MgB}_{2}$ films exhibit considerably larger critical current density as compared to undoped $\mathrm{MgB}_{2}$ film. The enhanced $J_{\mathrm{c}}$ could be attributed to the strong flux pinning achieved by the incorporation of $\mathrm{C}$ into the $\mathrm{MgB}_{2}$ as well as by the high density of grain boundaries. These results imply that the HFA-HPCVD would be a promising technique to fabricate $\mathrm{C}$-doped $\mathrm{MgB}_{2}$ superconducting wires and tapes with high $J_{\mathrm{c}}$ values for large scale applications.

\section{ACKNOWLEDGMENT}

This work was supported by Mid-career Researcher Program through National Research Foundation of Korea (NRF) grant funded by the Ministry of Education, Science \& Technology (MEST) (No. 2010-0029136).

\section{REFERENCES}

[1] W. N. Kang, H. J. Kim, E. M. Choi, C. U. Jung and S. I. Lee, "MgB superconducting thin films with a transition temperature of 39 Kelvin," Science, vol. 292, pp. 1521, 2001.

[2] D. Larbalestier, A. Gurevich, D. M. Feldmann, A. Polyanskii, "High- $T_{\mathrm{c}}$ superconducting materials for electric power applications," Nature (London), vol. 414, pp. 368, 2001.

[3] Mahipal Ranot and W. N. Kang," $\mathrm{MgB}_{2}$ coated superconducting tapes with high critical current densities fabricated by hybrid physical-chemical vapor deposition," Curr. Appl. Phys., vol. 12, pp. $353,2012$.

[4] J. H. Kim et al., "Microscopic role of carbon on $\mathrm{MgB}_{2}$ wire for critical current density comparable to NbTi," NPG Asia Mater., vol. 4, pp. e3, 2012. 
[5] X. X. Xi,"MgB ${ }_{2}$ thin films," Supercond. Sci. Technol., vol. 22, pp. 043001, 2009.

[6] C. G. Zhuang et al., "Significant improvements of the high-field properties of carbon-doped $\mathrm{MgB}_{2}$ films by hot-filament-assisted hybrid physical-chemical vapor deposition using methane as the doping source," Supercond. Sci. Technol., vol. 21, pp. 082002, 2008.
[7] M. Ranot, W. K. Seong, Soon-Gil Jung, W. N. Kang, J. Joo, C-J Kim, B-H Jun, S Oh, "Effect of SiC-impurity layer and growth temperature on $\mathrm{MgB}_{2}$ superconducting tapes fabricated by HPCVD," Chem.Vapor. Depos., vol. 18, pp. 36, 2012.

[8] S. M. Kazakov et al., "Carbon substitution in $\mathrm{MgB}_{2}$ single crystals: structural and superconducting properties," Phys. Rev. B, vol. 71, pp. 024533, 2005. 